\title{
PGC-1 $\alpha$ is responsible for survival of multiple myeloma cells under hyperglycemia and chemotherapy
}

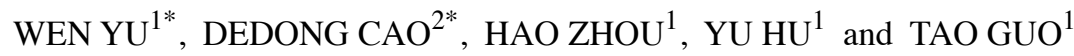 \\ ${ }^{1}$ Institute of Hematology, Union Hospital, Tongji Medical College, Huazhong University of Science and Technology; \\ ${ }^{2}$ Institute of Cancer, Renmin Hospital of Wuhan University, Wuhan University, Wuhan, Hubei 430000, P.R. China
}

Received December 8, 2014; Accepted February 5, 2015

DOI: 10.3892/or.2015.3809

\begin{abstract}
The association between hyperglycemia and outcomes during chemotherapy has been reported in several tumors, including multiple myeloma (MM). However, the underlying mechanism of how hyperglycemia affects the survival of MM cells during chemotherapy remain to be elucidated. MM cells were cultured in $10 \mathrm{mM}$ glucose with or without chemotherapeutic agents. Following treatment of MM cells with dexamethasone or bortezomib, an MTT assay was used to evaluate the toxicity of dexamethasone or bortezomib on cell proliferation, and changes of reactive oxygen species (ROS) level were detected by flow cytometry (FCM) analysis. Small interference RNA (siRNA) was applied to inhibit the expression of peroxisome proliferator-activated receptor $\gamma$ coactivator- $1 \alpha$ (PGC-1 $\alpha$ ). Expressions of PGC-1 $\alpha$ and antioxidant factors such as superoxide dismutase 2 (SOD-2), glutathione peroxidase 1 (GPX-1), and catalase (CAT) were measured by RT-PCR prior to and following treatment. The results showed that the level of PGC-1 $\alpha$ in MM cells cultured in high-glucose medium was upregulated prior to and following treatment of chemotherapeutic agents, and these cells showed less fold-change of ROS after insult of drugs, when compared to the control. Genes encoding antioxidant factors such as SOD-2 and CAT were also upregulated. Inhibition of PGC-1 $\alpha$ enhanced the toxicity of antitumor agents, associated with reduced expression of antioxidant factors, and elevated level of ROS. The present findings suggested that hyperglycemia may influence the anticancer effect of chemotherapeutic agents in MM by upregulating the expression of PGC- $1 \alpha$ and associated antioxidant factors. Inhibition of PGC-1 $\alpha$ or control of
\end{abstract}

Correspondence to: Professor Tao Guo, Institute of Hematology, Union Hospital, Tongji Medical College, Huazhong University of Science and Technology, 1277 Jiefang Road, Wuhan, Hubei 430000, P.R. China

E-mail: guotao1968@163.com

*Contributed equally

Key words: hyperglycemia, PGC-1 $\alpha$, reactive oxygen species, bortezomib, multiple myeloma hyperglycemia may be beneficial in improving the efficacy of chemotherapy in MM patients with diabetes.

\section{Introduction}

Multiple myeloma (MM) is a malignant plasma-cell disorder characterized by the abnormal proliferation of plasma cells in the bone marrow microenvironment, monoclonal protein in blood or urine, and relevant organ dysfunction (1). MM accounts for $1 \%$ of all types of cancer and $13 \%$ of hematologic malignancies, and the incidence and prevalence of MM increases with age, thus the median age at diagnosis is 70 years (2). Consequently, MM is prevalent in the elderly, and pre-existing comorbidities are usually present at diagnosis, type 2 diabetes being the most important one as its incidence is on the increase worldwide, along with the aging of population (3). It has been established that the morbidity of pre-existing diabetes in elderly patients with MM is $\sim 6-11 \%$ (3). Besides pre-existing diabetes, application of dexamethasone may cause steroidinduced diabetes (4), contributing to the increased prevalence of diabetes in elderly MM patients. Thus, more attention should be focused on the treatment of MM patients with diabetes.

The impact of pre-existing diabetes or steroid-induced diabetes on patients with MM has been suggested in a few clinical studies (3,5-7). Diabetes is associated with increased risks of adverse events such as peripheral neuropathy and venous thrombosis in MM patients who received bortezomib, thalidomide or lenalidomide (3). In addition, another study published by Jung et al (5) evaluated the incidence of hyperglycemia during induction therapy in $155 \mathrm{MM}$ patients and its effect on serious infections during that period of treatment. Those authors found that $12.9 \%$ of the patients developed hyperglycemia ( $\geq 200 \mathrm{mg} / \mathrm{dl}$ ) and serious infections occurred in $18.1 \%$ of this population, while infection-associated mortality occurred in one patient (5). These adverse events may influence the integrity of the treatment. Regarding the clinical outcomes, diabetes was proven to be an indicator of poor prognosis. Chou et al enrolled $310 \mathrm{MM}$ patients who were identified with pre-existing diabetes (3). Compared with their non-diabetic counterparts, MM patients with diabetes had a significantly higher proportion of renal dysfunction, advanced clinical staging, and higher all-cause mortality risk $(\mathrm{HR}=1.509, \mathrm{P}=0.037)$. In accordance with this result, Wu et al found that diabetes, steroid-induced diabetes in particular, is 
associated with poor clinical outcomes in MM (7). The highest level of post-load glycemia was associated with an evaluated risk of mortality in $\mathrm{MM}(\mathrm{HR}=3.06, \mathrm{P}<0.05)$ in another study by Chiu et al (8). These findings demonstrated the unfavorable impact of pre-existing and steroid-induced diabetes on clinical features and OS in patients with MM. However, little is known regarding how diabetes or hyperglycemia affects the efficacy of treatment for patients with MM.

Hyperglycemia influences the biological activities in cells and tissues. Several biochemical pathways have been proven to be closely associated with hyperglycemia, one of the best known pathways is high-glucose-mediated upregulation of reactive oxygen species $(\operatorname{ROS})(9,10)$. ROS are the by-products of cellular metabolism, and mitochondria are the main sources of ROS (11). Various antioxidant systems for removing ROS exist in cells. In general, the generation and clearance of ROS are maintained in the dynamic balance. When the production of ROS is out of control, it usually leads to oxidative stress, even apoptosis $(12,13)$. Normal cells in a high-glucose environment usually generate excessive ROS, leading to cell damage $(14,15)$. It was hypothesized that hyperglycemia-induced ROS was useful in improving the efficacy of anti-myeloma agents such as bortezomib or dexamethasone. However, the existing clinical evidence mentioned above did not support this hypothesis. Thus, the cause for the impaired anticancer effect of chemotherapy on MM with hyperglycemia remains to be determined.

Peroxisome proliferator-activated receptor $\gamma$ coactivator- $1 \alpha$ (PGC-1 $\alpha)$ is a transcriptional coactivator of nuclear receptors and plays critical roles in various metabolic pathways, including oxidative phosphorylation, fatty acid oxidation, glucose metabolism, and cellular respiration (16). PGC-1 $\alpha$ has been well studied in diabetes and exercise, however, little is known concerning the role of PGC-1 $\alpha$ in MM. In a previously published study, we found that PGC-1 $\alpha$ was upregulated in MM cells and was associated with glucose metabolism and angiogenesis (17). It has been suggested that PGC-1 $\alpha$ induces several key ROS-detoxifying enzymes when cells experience hyperglycemia and oxidative stress (18-21). However, it is not clear whether PGC-1 $\alpha$ is involved in regulating ROS in MM under hyperglycemic conditions and thus affecting the efficacy of chemotherapy.

Based on the observations that diabetes affects MM response to drugs and that hyperglycemia may interfere with the cellular production of ROS in MM, we investigated whether hyperglycemia influenced the axis PGC- $1 \alpha$-ROS-antioxidants in the setting of the increased level of glucose. For this purpose, we cultured MM cells in $10 \mathrm{mM}$ glucose culture medium, and then treated these cells with bortezomib. Our study provided in vitro evidence to explain the reason for MM patients with high-glucose condition suffering poor outcome when compared with non-hyperglycemic MM patients, and suggested that PGC-1 $\alpha$ is a potential target for improving the efficacy of treatment in MM patients with diabetes or steroid-induced diabetes.

\section{Materials and methods}

Cell culture. RPMI-8226, U266 and ARH77 MM cell lines were purchased from the American Type Culture Collection (Manassas, VA, USA). The cells were cultured in RPMI-1640 medium (HyClone, Thermo Fisher Scientific, Rockford, IL, USA) containing $10 \%$ fetal bovine serum and $100 \mathrm{U} / \mathrm{ml}$ penicillin/streptomycin, and were incubated at $37^{\circ} \mathrm{C}$ under $5 \% \mathrm{CO}_{2}$ conditions. To create a hyperglycemic condition in vitro, an additional $625 \mathrm{mg}$ of glucose were supplemented into $500 \mathrm{ml}$ ordinary RPMI-1640 medium. Bortezomib (kindly provided by Professor $\mathrm{Yu} \mathrm{Hu}$ ) and dexamethasone (Sigma-Aldrich, St. Louis, MO, USA) were used as chemotherapeutic agents to treat MM cells.

Small-interference RNA (siRNA). To inhibit the expression of PGC-1 $\alpha$, we introduced the siRNA-targeting PGC- $1 \alpha$ which was designed and produced by GenePharma (GenePharma, Suzhou, China). Briefly, MM cells were treated with the Lipofectamine $2000^{\mathrm{TM}}$ reagent (Invitrogen, Carlsbad, CA, USA) according to the manufacturer's instructions. MM cells were collected at $24 \mathrm{~h}$ after transfection. The sequence information for siRNA is presented as: siRNA for GAPDH, sense and antisense control DNA (provided with the kit); siRNA for PGC-1 $\alpha, 5$ '-GCCAAACCAACAACUUUAUUU-3' (forward) and 5'-AUAAAGUUGUUGGUUUGGCUU-3' (reverse).

MTT assay. MM cells cultured in normal or hyperglycemic conditions were seeded in 96-well plates at a density of $3.5-5 \times 10^{4}$ cells/well. Subsequent to treatment with different doses of chemotherapeutic agents including bortezomib and dexamethasone for $24 \mathrm{~h}, 10 \mu \mathrm{l}$ MTT [3-(4,5-dimethylthiazolyl-2)-2,5-diphenyltetrazolium bromide] solution was added to each well. The plates were then incubated for $4 \mathrm{~h}$ in the dark and detergent reagent was added until the purple formazan was completely dissolved. The assay was performed by a microplate reader (Bio-Rad, La Jolla, CA, USA) and the absorbance at $490 \mathrm{~nm}$ was measured and recorded. The inhibition rate of proliferation was defined as: Inhibition rate $=\left(\mathrm{OD}_{\text {control }}-\mathrm{OD}_{\mathrm{drug}}\right.$ - $\left.\mathrm{OD}_{\text {background }}\right) /\left(\mathrm{OD}_{\text {control }}\right.$ - $\left.\mathrm{OD}_{\text {background }}\right) \times 100 \%$.

cDNA synthesis and quantitative PCR analysis. The procedures were performed according to previously described methods (17). Total RNAs in MM cells were extracted by using the TRIzol reagent (Invitrogen). cDNA was then synthesized using a FSQ-101 cDNA Reverse Transcription kit (Toyobo, Life Science Department, Osaka, Japan). Quantitative RT-PCR (RT-qPCR) was performed using the SYBR-Green PCR system on a Life ABI 7500 instrument (Applied Biosystems, Foster City, CA, USA), and the results were normalized to $\beta$-actin. Primer sequences used were: $\beta$-actin: 5'-TTC CAG CCT TCC TTC CTG G-3' (forward) and 5'-TTG CGCTCAGGAGCAAT-3' (reverse);PGC-1 $\alpha$ :5'-TGGTGCCAC CACCATCAAAGA-3' (forward) and 5'-TCACCAAACAGC CGCAGACTG-3' (reverse); catalase (CAT): 5'-CCAGTCGGT GTATGCCTTCT-3' (forward) and 5'-GGACGCCACATTCTC GATAAG-3' (reverse); glutathione peroxidase-1: 5'-CCA GTCGGTGTATGCCTTCT-3' (forward) and 5'-GGACGC CACATTCTCGATAAG-3' (reverse); glutathione peroxidase-4: 5'-GCTGTGGAAGTGGATGAA-3' (forward) and 5'-GATGAGGAACTGTGGAGAG-3' (reverse); superoxide dismutase-1: 5'-ACTCATCTGTTATCCTGCTAG-3' (forward) and 5'-GCCTCATAATAAGTGCCA TAC-3' (reverse); superoxide dismutase-2: 5'-TCACCGAGGAGAAGTACC-3' (forward) and 5'-TTGATATGACCACCACCATT-3' (reverse). 

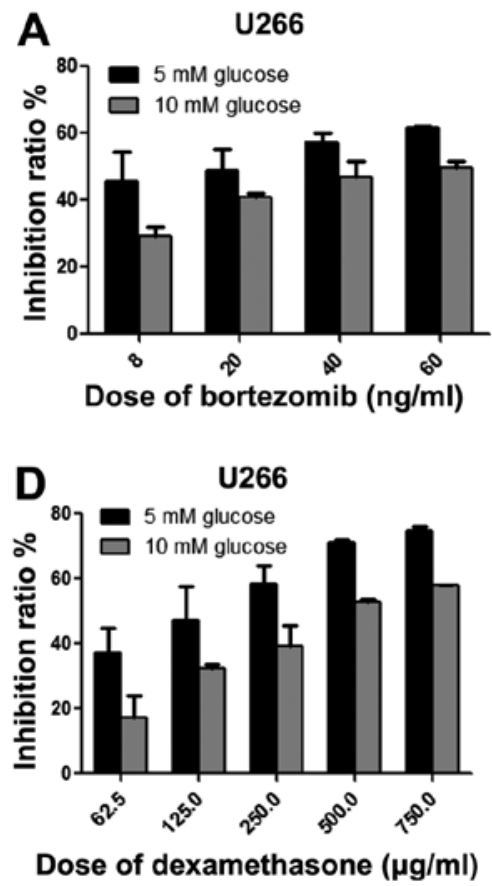
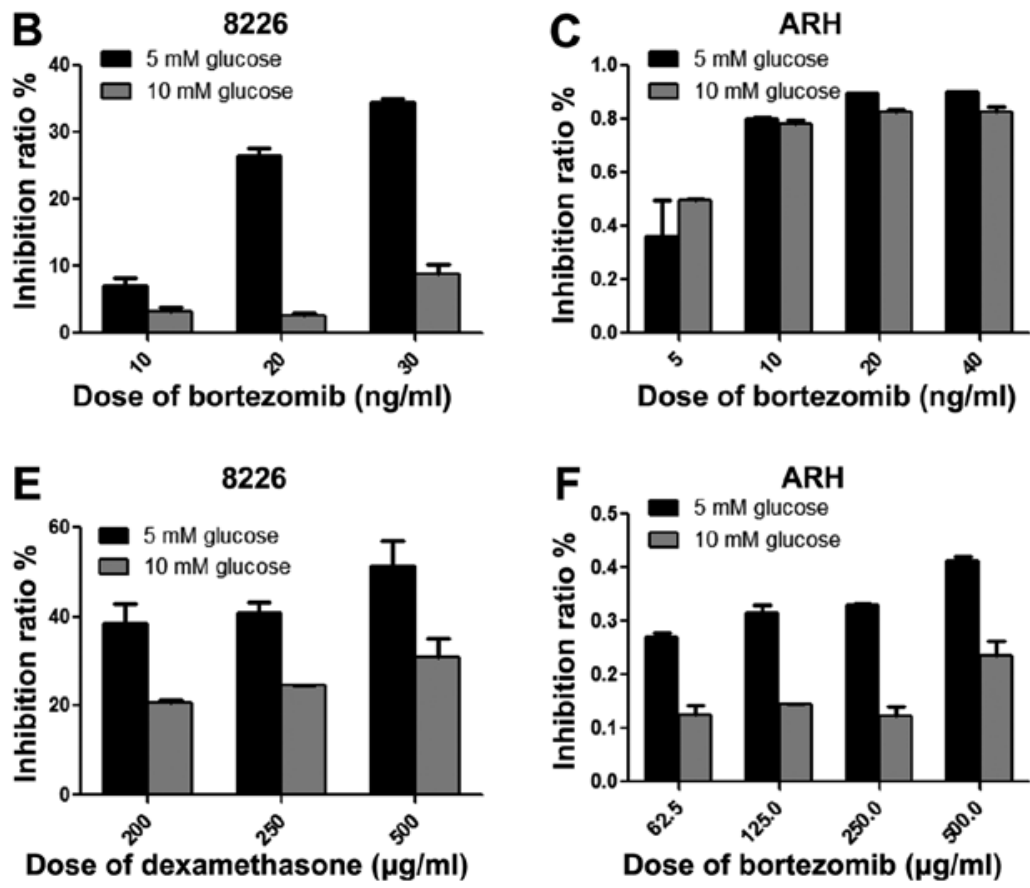

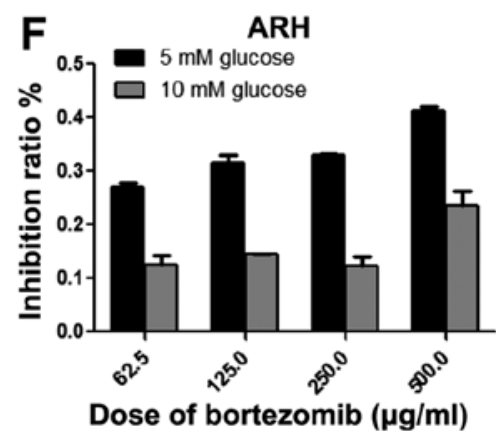

Figure 1. Proliferation inhibitory rates of MM RPMI-8226, ARH77 and U266 cells after treatment. Following treatment with different doses of bortezomib or dexamethasone, the relative proliferation rates of U266 (A and D), RPMI-8226 (B and E) and ARH77 (C and F) cells were examined. Hyperglycemia differentially attenuated the cellular toxicity of chemotherapeutic agents in MM cells. MM, multiple myeloma.
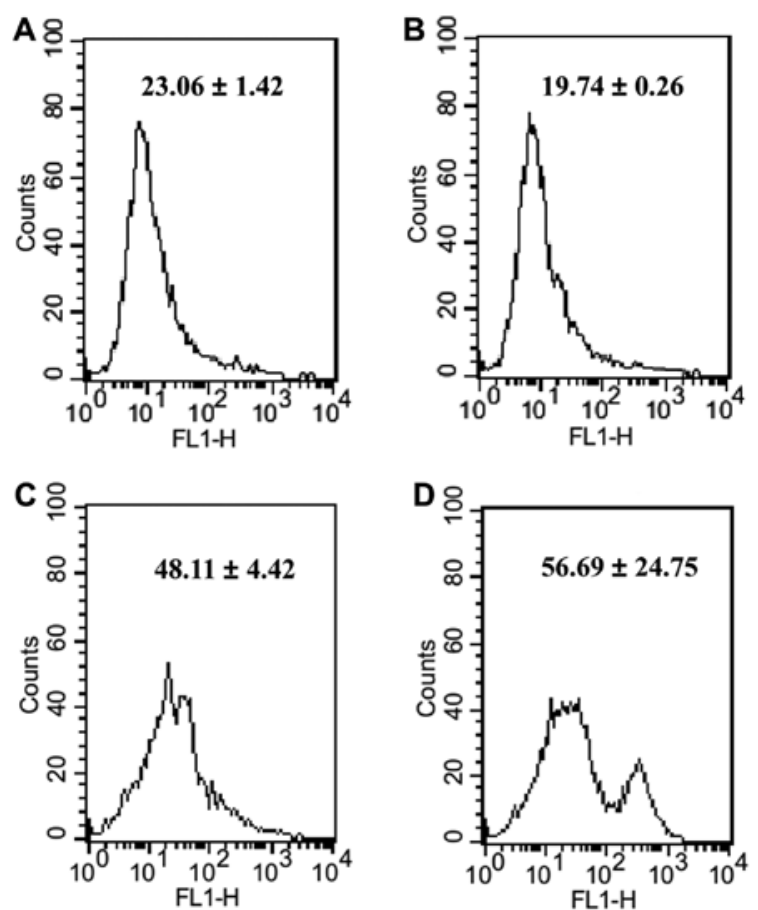

Figure 2. Levels of reactive oxygen species (ROS) in RPMI-8226 cells before and after treatment of bortezomib under normal or high-glucose condition. RPMI-8226 cells cultured in high glucose have a similar level of ROS, when compared with the control. When using bortezomib $(10 \mathrm{ng} / \mathrm{ml})$ to treat these cells, elevated ROS were tested regardless of glucose concentration. However, the fold change of ROS in high glucose condition is a little higher than that of control. Level of ROS in (A) normal condition, (B) high glucose condition, (C) normal condition and chemotherapy insult, (D) high-glucose condition and chemotherapy insult.

ROS. Briefly, MM cells cultured in normal or high glucose were collected and incubated with $10 \mu \mathrm{M}$ DCFH-DA
(Sigma-Aldrich) for $30 \mathrm{~min}$ at $37^{\circ} \mathrm{C}$. The suspended cells were rotated gently every $5 \mathrm{~min}$ during the period of incubation. The cells were then washed and resuspended in PBS. The mean fluorescence intensity of DCF was detected by the FACSCalibur flow cytometer and analyzed using Cell Quest software (both from BD Biosciences, San Jose, CA, USA).

Western blotting. Whole cell lysate as well as SDS-PAGE, electrophoretic transfer and immunoblotting were prepared as previously described (22). Protein samples were analyzed using chemiluminescence detection (ECL; Amersham Pharmacia, Buckinghamshire, UK, GB). Antibodies used in this section were purchased from Cell Signaling Technology. Total protein concentration was measured according to the manufacturer's instructions (Thermo Scientific Pierce, Rockford, IL, USA).

Statistical analysis. SPSS 15.0 software (SPSS Inc., Chicago, IL, USA) was used to analyze all recorded data. Data are presented as means \pm SD values. Differences between groups were analyzed by ANOVA or Student's t-test and were considered to be statistically significant if $\mathrm{P}<0.05$. Each experiment was repeated at least three times, independently.

\section{Results}

Hyperglycemia decreases the toxicity of chemotherapeutic agents. Human RPMI-8226, U266 and ARH-77 MM cells were cultured in hyperglycemic conditions continuously, and treated with different chemotherapeutic agents including bortezomib and dexamethasone independently. After $24 \mathrm{~h}$ of treatment, we examined the toxicity of bortezomib and dexamethasone in RPMI-8226, U266 and ARH77 by MTT. As shown in Fig. 1, MM RPMI-8226, U266 and ARH77 cells 

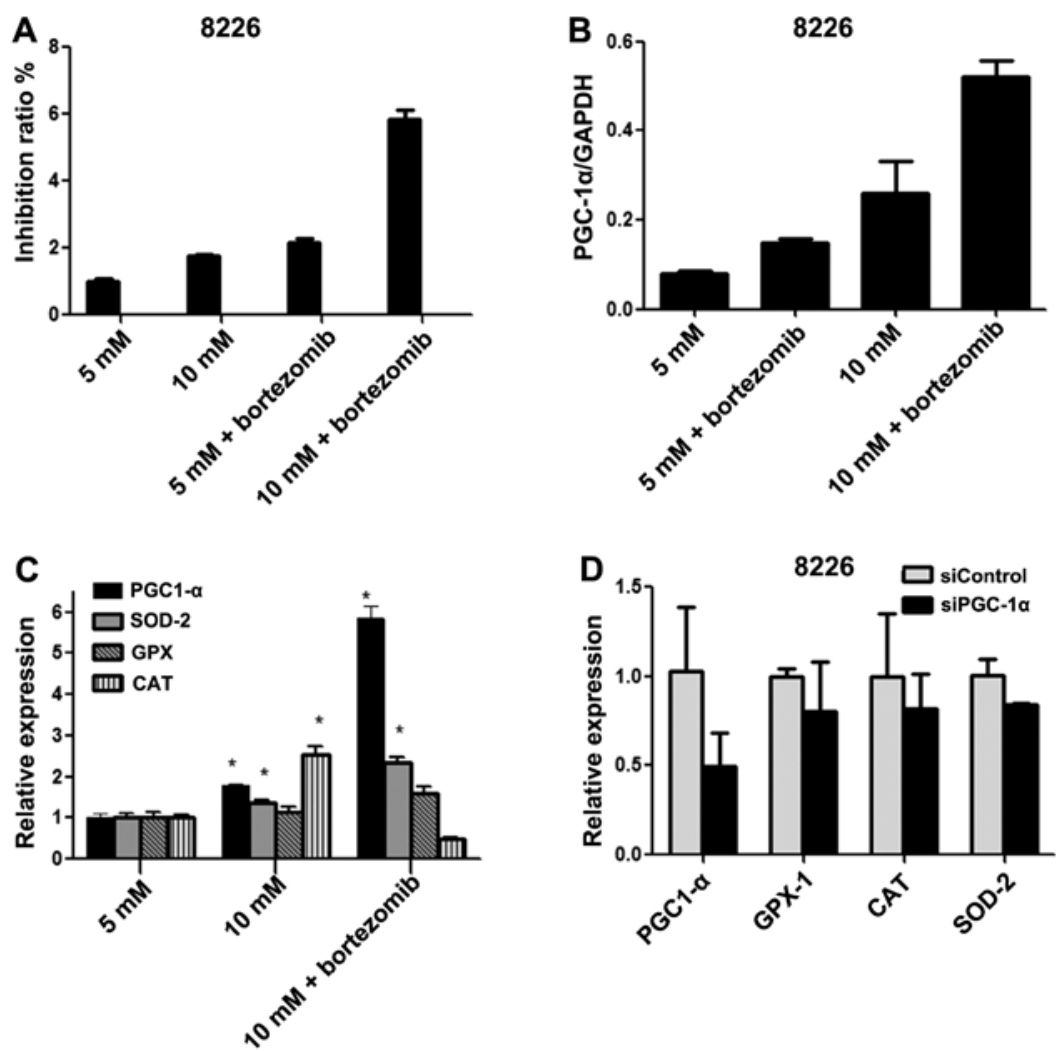

Figure 3. The expression of PGC-1 $\alpha$ and related antioxidant factors after chemotherapy or siRNA treatment. After treating RPMI-8226 cells with bortezomib or siRNA, the relative expression of PGC-1 $\alpha$, CAT, GPX-1 and SOD-2 are measured. (A, B and C) Suppression of PGC-1 $\alpha$ by siRNA resulted in a decreased expression of PGC-1 $\alpha$, CAT, GPX-1 and SOD-2. (A and B) Expression of PGC-1 $\alpha$ in RPMI-8226 cells under normal glucose, high-glucose and chemotherapy conditions. (C) Expression of PGC-1 $\alpha$, CAT, GPX-1 and SOD-2 under normal glucose, high-glucose and chemotherapy conditions, when compared to normal-glucose condition. (D) The expression of PGC-1 $\alpha$, CAT, GPX-1 and SOD-2 in RPMI-8226 cells were inhibited after treating cells with siPGC-1 $\alpha$, when compared to siControl. MM, multiple, myeloma; SOD, superoxide dismutase; GPX, glutathione peroxidase; CAT, catalase; Dex, dexamethasone; Bor, bortezomib; Met, metformin; PGC-1 $\alpha$, peroxisome proliferator-activated receptor $\gamma$ coactivator- $1 \alpha$.

cultured in high glucose showed decreased toxicity of bortezomib. The inhibitory rates of bortezomib at the same dose were much lower than those in cells grown in normal glucose conditions. These data indicated that hyperglycemia reduced the efficacy of chemotherapeutic agents. This is in accordance with the results from studies showing that elevated blood glucose levels are associated with a poor outcome in cancer patients and hyperglycemia is a prognostic factor with higher risk of death in lung and breast cancer patients $(23,24)$. In addition, $20 \mathrm{mM}$ glucose treatment of U266, ARH77 and other cell lines caused a decrease of toxicity of dexamethasome that may contribute to the resistance of chemotherapy (25).

We examined the level of ROS in MM cells which were incubated in high-glucose culture medium, before and after treatment of bortezomib. The cellular ROS level was labeled by CM-H2DCFDA and measured using a FACSCalibur flow cytometer. As shown in Fig. 2, following treatment of chemotherapeutic agents, we observed that the ROS level in MM RPMI-8226 cells cultured in high-glucose culture medium were a little higher than that of control. The fold-change of ROS in the cells treated with bortezomib and $10 \mathrm{mM}$ glucose was $>2$ when compared with $10 \mathrm{mM}$ glucose alone.

PGC-1 $\alpha$ and relevant antioxidant factors are upregulated in MM cells treated with chemotherapeutic agents under hyperglycemic conditions. Levels of PGC-1 $\alpha$ are highly regulated by cellular stress and therefore allow metabolic adaptation (26). Thus, we assessed the impact of high glucose on the expression of PGC-1 $\alpha$ in the RPMI-8226 MM cell line. We measured the relative expression of PGC- $1 \alpha$ mRNA using RT-PCR. As shown in Fig. 3, levels of PGC-1 $\alpha$ mRNA in cells cultured in $10 \mathrm{mM}$ glucose condition were higher than those cultured in normal medium. We assessed PGC-1 $\alpha$ expression in cells treated with bortezomib, and found that levels of PGC-1 $\alpha$ were further increased when compared with cells without treatment of bortezomib, especially in cells grown in $10 \mathrm{mM}$ glucose medium.

The levels of PGC-1 $\alpha$ protein in RPMI-8226 cells were assessed by western blot analysis. We used metformin, an AMPK activator, to induce the expression of PGC- $1 \alpha$ in MM cells as a positive control. We found an increased expression of PGC-1 $\alpha$ protein in cells grown in $10 \mathrm{mM}$ glucose or treated with bortezomib when compared with the control group. These findings indicate that PGC- $1 \alpha$ is also highly expressed in $\mathrm{MM}$, as high glucose and chemotherapeutic agents induce the expression of PGC-1 $\alpha$ at the mRNA and protein levels.

PGC- $1 \alpha$ is known to be involved in the regulation of the antioxidant system. However, it is unclear whether PGC-1 $\alpha$ retains the same function in MM. Thus, we assessed the impact of PGC-1 $\alpha$ on the expression of a set of genes relating to antioxidant factors. Cells cultured in 5 or $10 \mathrm{mM}$ glucose and treated with or without $10 \mathrm{ng} / \mathrm{ml}$ bortezomib were collected, 

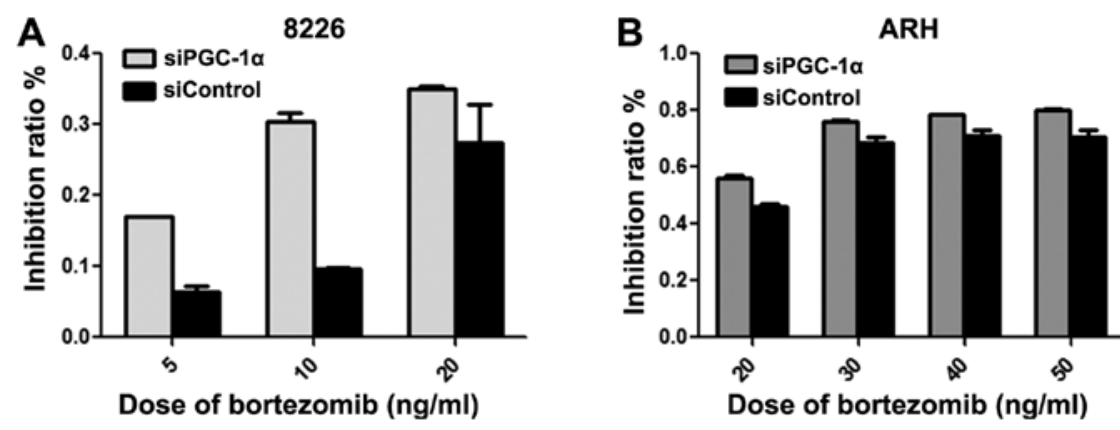

Figure 4. Inhibition of PGC-1 $\alpha$ enhances the accumulation of ROS during hyperglycemia and chemotherapy. After interfering the expression of PGC-1 $\alpha$ with siRNA, the levels of ROS in RPMI-8226 and ARH77 cells were upregulated, when compared with siControl. (A) RPMI-8226 cells were treated with siControl and bortezomib (10 ng/ml). (B) RPMI-8226 cells were treated with siPGC-1 $\alpha$ and bortezomib. (C) ARH77 cells were treated with siControl and bortezomib. (D) ARH77 cells were treated with siPGC-1 $\alpha$ and Bortezomib. PGC-1 $\alpha$, peroxisome proliferator-activated receptor $\gamma$ coactivator-1 $\alpha$; ROS, reactive oxygen species.
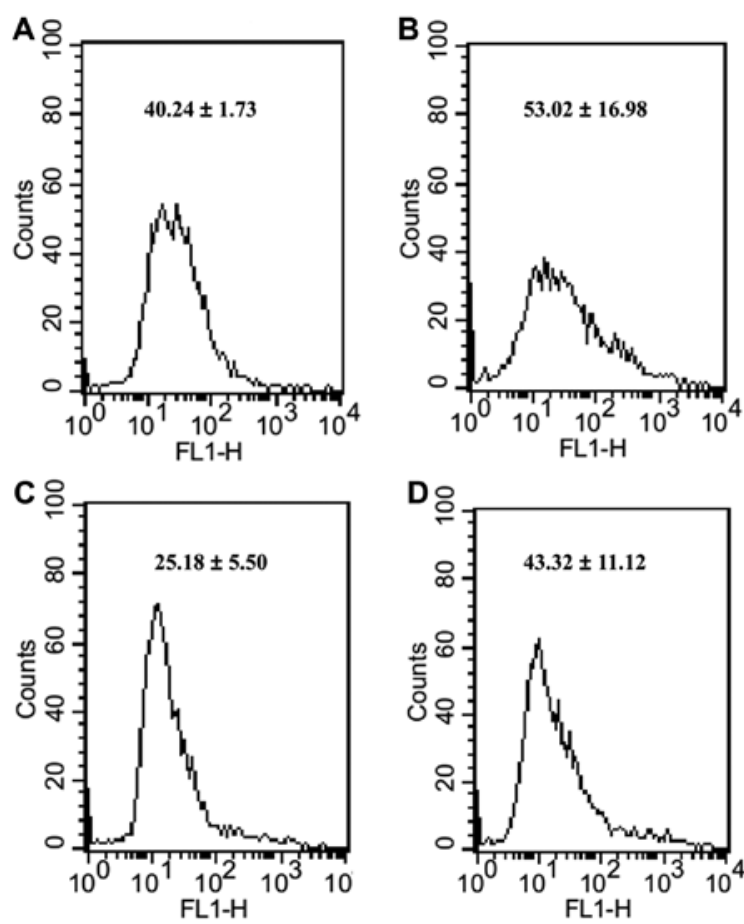

Figure 5. Inhibition of PGC-1 $\alpha$ enhances the accumulation of ROS in high glucose conditions. (A) RPMI-8226 cells were treated with siControl. (B) RPMI-8226 cells were treated with siPGC-1 $\alpha$. (C) ARH77 cells were treated with siControl. (D) ARH77 cells were treated with siPGC-1 $\alpha$.

and mRNA expressions of superoxide dismutase 2 (SOD-2), CAT and glutathione peroxidase 1 (GPX-1) in these cells were measured by RT-PCR. Increased expression of SOD-2 and GPX-1 was observed in cells treated with $10 \mathrm{mM}$ glucose and bortezomib (Fig. 3) when compared to the control, and these cells also showed an upregulated expression of PGC-1 $\alpha$.

Suppression of $P G C-1 \alpha$ by siRNA reverses the reduction of ROS and enhances the toxicity of chemotherapeutic agents in MM cells. To elucidate the role of PGC-1 $\alpha$ in the transcriptional regulation of antioxidant factors, we suppressed the expression of PGC- $1 \alpha$ in cells with siRNA targeting PGC- $1 \alpha$. As expected, the expression of PGC-1 $\alpha$ was reduced by PGC-1 $\alpha$ siRNA (Fig. 3D). Addtionally, the expression of SOD-2, CAT and GPX-1 were differentially reduced. However,
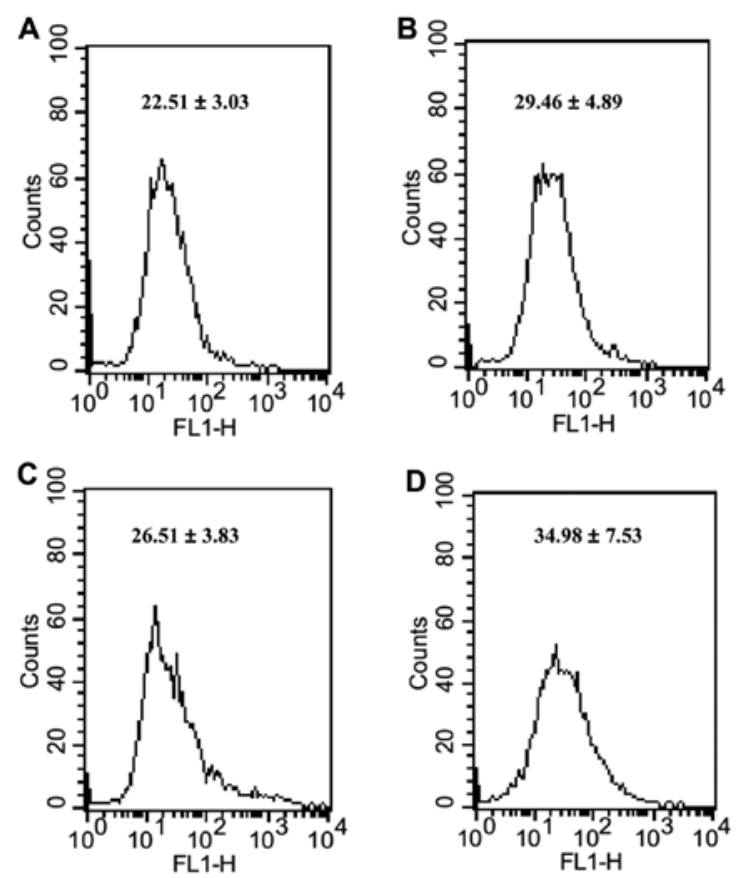

Figure 6. The cellular toxicity of bortezomib was enhanced under hyperglycemia by inhibiting PGC-1 $\alpha$. After inhibiting the level of PGC-1 $\alpha$ by siRNA, the proliferation inhibitory rates in RPMI-8226 and ARH77 cells treated with siControl was differentially lower than that in cells treated with siPGC-1 $\alpha$, at different doses of bortezomib. (A) Inhibition rates of proliferation in RPMI-8226 cells after siRNA treatment. (B) Inhibition rates of proliferation in ARH77 cells after siRNA treatment. PGC-1 $\alpha$, peroxisome proliferator-activated receptor $\gamma$ coactivator- $1 \alpha$.

the levels of SOD-1, GPX-4 and PRDX-1 were not affected by the suppression of PGC-1 $\alpha$ (data not shown), indicating that other pathways may exist that are independent of regulation by PGC-1 $\alpha$ during hyperglycemia.

To determine the effect of inhibition of PGC-1 $\alpha$ on the amount of ROS and toxicity of bortezomib, we tested cellular ROS level by FACSCalibur flow cytometer and toxicity of bortezomib by MTT in cells transfected with siPGC-1 $\alpha$. The level of ROS was increased (Figs. 4 and 5) and toxicity of bortezomib was enhanced (Fig. 6) when compared with the siControl group, suggesting that PGC- $1 \alpha$ may induce the reduced ROS level and toxicity of bortezomib regardless of 
hyperglycemia, thus targeting PGC-1 $\alpha$ may be beneficial for MM patients with or without evaluated blood-glucose level.

\section{Discussion}

The impact of hyperglycemia on the therapeutic effect of chemotherapeutic agents in MM, as well as how hyperglycemia influences outcomes of MM during treatment, remains poorly understood $(6,25,27)$. In the present study, we found that the cellular toxicity of bortezomib was reduced when treating MM cells cultured under high-glucose condition, accompanied with an increased expression of PGC-1 $\alpha$ and antioxidant factors such as SOD-2, CAT and GPX-1. The levels of ROS in these MM cells were not significantly increased following treatment with the agents, but a little decrease in ROS levels. However, following treatment of cells with siPGC-1 $\alpha$, the accumulation of ROS and the efficacy of chemotherapy agents were enhanced in MM cells. Our results suggested that hyperglycemia did not enhance or decrease the tumor-killing effect of chemotherapeutic agents, and the upregulated PGC- $1 \alpha$ and associated antioxidant factors likely induced the indistinctive elevation of ROS in MM cells cultured under high-glucose condition.

MM cells grown under high-glucose conditions readily obtain more glucose as abnormal glucose uptake and metabolism exist in MM, as identified in a PET-CT examination (28). Glucose is an important substance for proliferation and survival of normal and malignant cells (29-31). Glucose is mainly used to produce energy material such as ATP, and can provide synthetic intermediates such as lipid and carbon skeleton for cell proliferation. Studies have shown that glucose participates in the regulation of a variety of key factors associated with cell apoptosis, such as Mcl-1 and Bcl-2 (32-35). In addition, glucose is capable of preventing the release of cytochrome $c$ and subsequent cell apoptosis by maintaining the interaction of mitochondria and hexokinase II (36). In the present study, we found that MM cells under hyperglycemia had a lower proportion of apoptosis following treatment with chemotherapeutic agents, but a higher level of PGC-1 $\alpha$, when compared with MM cells cultured at the normal level of glucose. PGC-1 $\alpha$ has been proved to be involved in the regulation of glucose metabolism, and is a master regulator of GLUT-4, which is an important glucose transporter in MM. We have previously demonstrated that PGC-1 $\alpha$ has the ability of upregulating the expression of GLUT-4 in MM (17), and myeloma cells exhibit reliance on constitutively cell surfacelocalized GLUT-4 for basal glucose consumption, maintenance of Mcl-1 expression, growth, and survival (28). Elevated glucose uptake and metabolism enables MM cells to possess the advantages of proliferation and anti-apoptosis mentioned above. Besides, intracellular glucose can be used to generate intermediates such as NADPH, and exert a fundamental role in maintaining the integrity of the mitochondria (37). Molecules such as NADPH are especially important for salvaging the oxidative stress and reducing insult-associated-excessive ROS in cells. This beneficial regulation allows the cells to enhance the ability to preserve themselves from the damage of excessive ROS. Based on these settings, hyperglycemia induced the expression of PGC- $1 \alpha$ and associated antioxidant factors (SOD-2, CAT and GPX-1), leading to the relatively decreasing of ROS in MM cells, and eventually resulting in reduced cellular toxicity of the chemotherapeutic agents. This is in accordance with the results of Friday et al who have reported that hyperglycemia increased the $\mathrm{IC}_{50}$ value of dexamethasone in MM cells (25).

Apart from hyperglycemia induced by drugs, type 2 diabetes disease is present in $\sim 10 \%$ of patients diagnosed as MM, and these patients had a significantly higher mortality risk compared with non-diabetic ones $(3,4,6)$. Therefore, hyperglycemia should be positively and effectively controlled in order to improve survival outcomes. In a study conducted by Vu et al it was shown that, the administration of metformin and thiazolidinediones may be associated with improved outcomes in acute lymphoblastic leukemia patients with hyperglycemia (38). Our results have demonstrated that high glucose attenuates the antitumor effect of chemotherapeutic agents by inducing the expression of PGC-1 $\alpha$ and antioxidant factors. Although the treatment of patients with MM and pre-existing diabetes or drug-induced hyperglycemia forms a complex challenge for physicians, these findings suggest that it is essential to treat diabetes and hyperglycemia to improve treatment outcomes.

PGC-1 $\alpha$ is a transcriptional coactivator initially recognized as a functional activator of the peroxisome proliferator-activated receptor (PPAR)- $\gamma$ in brown adipose tissue (39) and recently identified as an important regulator of numerous aspects of metabolism by interacting with nuclear receptors and transcription factors to activate the transcription of their target genes (40-42). In recent years, studies have determined that PGC- $1 \alpha$ has a regulatory mechanism for the expression of endogenous antioxidant factors (40). In skeletal muscle from PGC-1 $\alpha$ knockout (KO) mice, reduced mRNA expression of SOD-1, SOD-2, and/or GPX-1 was observed when compared to wild-type mice, while PGC-1 $\alpha$ overexpression showed an upregulation of SOD-2 (40). PGC-1 $\alpha$ knockout fibroblasts showed that a decrease in mRNA levels of SOD-2, CAT, and GPX-1 compared to wild-type fibroblasts and mice with loss of PGC-1 $\alpha$ were more vulnerable to oxidative stress (18). In addition, $\mathrm{PGC}-1 \alpha$ may increase the uncoupling capacity and concomitantly reduce mitochondrial ROS production. Furthermore, PGC-1 $\alpha$ promotes the expression of SIRT-3 by interacting with an ER- $\alpha$ binding element mapped to the SIRT3 promoter region. SIRT3 can activate SOD-2 through a post-translational mechanism (40). Taken together, the results show that, PGC-1 $\alpha$ has a role in reducing the expression and activity of ROS damage by upregulating antioxidant factors. As PGC- $1 \alpha$ exerts its roles by modulating the metabolism of non-malignant cells, the role of PGC- $1 \alpha$ in tumors is important (43-45). In our study, cells cultured under high-glucose conditions showed a higher level of PGC-1 $\alpha$, and these cells showed a higher level of SOD-2, CAT and GPX-1 during chemotherapy than cells cultured in ordinary RPMI-1640 medium, supporting the hypothesis that PGC- $1 \alpha$ maintains the role of regulating antioxidant factors in MM. Furthermore, we suppressed the expression of PGC- $1 \alpha$ by siRNA and these cells showed a decreased expression of SOD-2, CAT and GPX-1, accompanied with an increased ROS and enhanced toxicity of chemotherapeutic agents. Thus, PGC- $1 \alpha$ should be responsible for the decreased efficacy of chemotherapeutic agents in MM, and it may be effective to improve efficacy by targeting PGC-1 $\alpha$. 
In conclusion, though this is an in vitro study and there are several limitations, our study is sufficient to show that hyperglycemia attenuates the anti-MM effect of chemotherapeutic agents by upregulating PGC- $1 \alpha$ and related antioxidant factors, leading to the reduction of ROS. Treatment of hyperglycemia holds may be benefiical for patients with MM and diabetes or drugs induced hyperglycemia. Targeting PGC-1 $\alpha$ may be sufficient in improving the efficacy of chemotherapy in cancer treatment.

\section{References}

1. Guglielmelli T and Palumbo A: Incorporating novel agents in the management of elderly myeloma patients. Curr Hematol Malig Rep 8: 261-269, 2013.

2. Palumbo A and Mina R: Management of older adults with multiple myeloma. Blood Rev 27: 133-142, 2013.

3. Chou YS, Yang CF, Chen HS, et al: Pre-existing diabetes mellitus in patients with multiple myeloma. Eur J Haematol 89: 320-327, 2012.

4. Issa ZA, Zantout MS and Azar ST: Multiple myeloma and diabetes. ISRN Endocrinol 2011: 815013, 2011.

5. Jung SH, Jang HC, Lee SS, et al: The impact of hyperglycemia on risk of severe infections during early period of induction therapy in patients with newly diagnosed multiple myeloma. Biomed Res Int 2014: 413149, 2014.

6. Ahmed YA and Eltayeb A: Clinical challenges: myeloma and concomitant type 2 diabetes. Int J Hematol Oncol Stem Cell Res 7: 34-41, 2013.

7. Wu W, Merriman K, Nabaah A, et al: The association of diabetes and anti-diabetic medications with clinical outcomes in multiple myeloma. Br J Cancer 111: 628-636, 2014.

8. Chiu BC, Gapstur SM, Greenland P, Wang R and Dyer A: Body mass index, abnormal glucose metabolism, and mortality from hematopoietic cancer. Cancer Epidemiol Biomarkers Prev 15 2348-2354, 2006.

9. Baynes JW: Role of oxidative stress in development of complications in diabetes. Diabetes 40: 405-412, 1991.

10. Busik JV, Mohr S and Grant MB: Hyperglycemia-induced reactive oxygen species toxicity to endothelial cells is dependent on paracrine mediators. Diabetes 57: 1952-1965, 2008.

11. Zorov DB, Juhaszova M and Sollott SJ: Mitochondrial reactive oxygen species (ROS) and ROS-induced ROS release. Physiol Rev 94: 909-950, 2014.

12. Edeas M: Strategies to target mitochondria and oxidative stress by antioxidants: key points and perspectives. Pharm Res 28 2771-2779, 2011

13. Vurusaner B, Poli G and Basaga $\mathrm{H}$ : Tumor suppressor genes and ROS: complex networks of interactions. Free Radic Biol Med 52: 7-18, 2012.

14. Valle I, Alvarez-Barrientos A, Arza E, Lamas S and Monsalve M: PGC-1alpha regulates the mitochondrial antioxidant defense system in vascular endothelial cells. Cardiovasc Res 66: 562-573, 2005.

15. Nishikawa T, Edelstein D, Du XL, et al: Normalizing mitochondrial superoxide production blocks three pathways of hyperglycaemic damage. Nature 404: 787-790, 2000.

16. Finck BN and Kelly DP: PGC-1 coactivators: inducible regulators of energy metabolism in health and disease. J Clin Invest 116: 615-622, 2006.

17. Cao D, Zhou H,Zhao J, et al: PGC-1 $\alpha$ integrates glucose metabolism and angiogenesis in multiple myeloma cells by regulating VEGF and GLUT-4. Oncol Rep 31: 1205-1210, 2014.

18. St-Pierre J, Drori S, Uldry M, et al: Suppression of reactive oxygen species and neurodegeneration by the PGC-1 transcriptional coactivators. Cell 127: 397-408, 2006.

19. Kong X, Wang R, Xue Y, et al: Sirtuin 3, a new target of PGC-1 alpha, plays an important role in the suppression of ROS and mitochondrial biogenesis. PLoS One 5: e11707, 2010.

20. Fujisawa K, Nishikawa T, Kukidome D, et al: TZDs reduce mitochondrial ROS production and enhance mitochondrial biogenesis. Biochem Biophys Res Commun 379: 43-48, 2009.

21. Zhang LN, Zhou HY, Fu YY, et al: Novel small-molecule PGC-1o transcriptional regulator with beneficial effects on diabetic $\mathrm{db} / \mathrm{db}$ mice. Diabetes 62: 1297-1307, 2013.
22. Pearce EL, Walsh MC, Cejas PJ, et al: Enhancing CD8 T-cell memory by modulating fatty acid metabolism. Nature 460: 103-107, 2009.

23. Villarreal-Garza C, Shaw-Dulin R, Lara-Medina F, et al: Impact of diabetes and hyperglycemia on survival in advanced breast cancer patients. Exp Diabetes Res 2012: 732027, 2012.

24. Luo J, Chen YJ and Chang LJ: Fasting blood glucose level and prognosis in non-small cell lung cancer (NSCLC) patients. Lung Cancer 76: 242-247, 2012.

25. Friday E, Ledet $\mathbf{J}$ and Turturro F: Response to dexamethasone is glucose-sensitive in multiple myeloma cell lines. J Exp Clin Cancer Res 30: 81, 2011.

26. Scarpulla RC, Vega RB and Kelly DP: Transcriptional integration of mitochondrial biogenesis. Trends Endocrinol Metab 23: 459-466, 2012.

27. Sheean PM, Kilkus JM, Liu D, Maciejewski J and Braunschweig CA: Incident hyperglycemia, parenteral nutrition administration and adverse outcomes in patients with myeloma admitted for initial auto-SCT. Bone Marrow Transplant 48: 1117-1122, 2013.

28. McBrayer SK, Cheng JC, Singhal S, Krett NL, Rosen ST and Shanmugam M: Multiple myeloma exhibits novel dependence on GLUT4, GLUT8, and GLUT11: implications for glucose transporter-directed therapy. Blood 119: 4686-4697, 2012.

29. Zhao Y, Butler EB and Tan M: Targeting cellular metabolism to improve cancer therapeutics. Cell Death Dis 4: e532, 2013.

30. Gitenay D, Wiel C, Lallet-Daher H, et al: Glucose metabolism and hexosamine pathway regulate oncogene-induced senescence. Cell Death Dis 5: e1089, 2014.

31. Zhao Y, Liu H, Riker AI, et al: Emerging metabolic targets in cancer therapy. Front Biosci (Landmark Ed) 16: 1844-1860, 2011.

32. DeBerardinis RJ, Mancuso A, Daikhin E, et al: Beyond aerobic glycolysis: transformed cells can engage in glutamine metabolism that exceeds the requirement for protein and nucleotide synthesis. Proc Natl Acad Sci USA 104: 19345-19350, 2007.

33. Zhao Y, Altman BJ, Coloff JL, et al: Glycogen synthase kinase 3alpha and 3beta mediate a glucose-sensitive antiapoptotic signaling pathway to stabilize Mcl-1. Mol Cell Biol 27: 4328-4339, 2007.

34. Danial NN, Gramm CF, Scorrano L, et al: BAD and glucokinase reside in a mitochondrial complex that integrates glycolysis and apoptosis. Nature 424: 952-956, 2003.

35. Rathmell JC, Fox CJ, Plas DR, Hammerman PS, Cinalli RM and Thompson CB: Akt-directed glucose metabolism can prevent Bax conformation change and promote growth factor-independent survival. Mol Cell Biol 23: 7315-7328, 2003.

36. Mathupala SP, Ko YH and Pedersen PL: Hexokinase II: cancer's double-edged sword acting as both facilitator and gatekeeper of malignancy when bound to mitochondria. Oncogene 25: 4777-4786, 2006

37. Gendron MC, Schrantz N, Metivier D, et al: Oxidation of pyridine nucleotides during Fas- and ceramide-induced apoptosis in Jurkat cells: correlation with changes in mitochondria, glutathione depletion, intracellular acidification and caspase 3 activation. Biochem J 353: 357-367, 2001.

38. Vu K, Busaidy N, Cabanillas ME, et al: A randomized controlled trial of an intensive insulin regimen in patients with hyperglycemic acute lymphoblastic leukemia. Clin Lymphoma Myeloma Leuk 12: 355-362, 2012.

39. Puigserver P, Wu Z, Park CW, Graves R, Wright $\mathrm{M}$ and Spiegelman BM: A cold-inducible coactivator of nuclear receptors linked to adaptive thermogenesis. Cell 92: 829-839, 1998.

40. Kang C and Li Ji L: Role of PGC-1 $\alpha$ signaling in skeletal muscle health and disease. Ann NY Acad Sci 1271: 110-117, 2012.

41. Radak Z, Zhao Z, Koltai E, Ohno H and Atalay M: Oxygen consumption and usage during physical exercise: the balance between oxidative stress and ROS-dependent adaptive signaling. Antioxid Redox Signal 18: 1208-1246, 2013.

42. Wenz T: Regulation of mitochondrial biogenesis and PGC-1a under cellular stress. Mitochondrion 13: 134-142, 2013.

43. Salem AF, Whitaker-Menezes D, Howell A, Sotgia F and Lisanti MP: Mitochondrial biogenesis in epithelial cancer cells promotes breast cancer tumor growth and confers autophagy resistance. Cell Cycle 11: 4174-4180, 2012.

44. Tennakoon JB, Shi Y, Han JJ, et al: Androgens regulate prostate cancer cell growth via an AMPK-PGC-1 $\alpha$-mediated metabolic switch. Oncogene 33: 5251-5261, 2014.

45. Girnun GD: The diverse role of the PPAR $\gamma$ coactivator 1 family of transcriptional coactivators in cancer. Semin Cell Dev Biol 23: 381-388, 2012. 